\title{
Correction to: Clinical and immunologic evaluation of three metastatic melanoma patients treated with autologous melanoma-reactive TCR- transduced T cells
}

\author{
Tamson Moore ${ }^{1}$ (D) . Courtney Regan Wagner ${ }^{2}$. Gina M. Scurti ${ }^{1} \cdot$ Kelli A. Hutchens ${ }^{3,4} \cdot$ Constantine Godellas $^{1}$. \\ Ann Lau Clark ${ }^{2}$. Elizabeth Motunrayo Kolawole ${ }^{5}$. Lance M. Hellman ${ }^{6} \cdot$ Nishant K. Singh $^{6} \cdot$ Fernando A. Huyke $^{6}$. \\ Siao-Yi Wang ${ }^{1} \cdot$ Kelly M. Calabrese ${ }^{1,7} \cdot$ Heather D. Embree $^{8} \cdot$ Rimas Orentas $^{8} \cdot$ Keisuke Shirai $^{9,10}$. \\ Emilia Dellacecca $^{3,11}$ - Elizabeth Garrett-Mayer ${ }^{9}$. Mingli Li $i^{9,12}$. Jonathan M. Eby ${ }^{3,11} \cdot$ Patrick J. Stiff ${ }^{2}$. \\ Brian D. Evavold ${ }^{5}$. Brian M. Baker ${ }^{6}$. I. Caroline Le Poole ${ }^{3,11,13}$ • Boro Dropulic ${ }^{8} \cdot$ Joseph I. Clark $^{2}$. \\ Michael I. Nishimura ${ }^{1}$
}

Published online: 20 December 2017

(c) Springer-Verlag GmbH Germany, part of Springer Nature 2017

\section{Correction to: Cancer Immunol Immunother https://doi.org/10.1007/s00262-017-2073-0}

The authors would like to make the following corrections to the published article:

Prior therapy for Patient 3 was high-dose IL-2, vemurafenib, ipilimumab, and dabrafenib (Table 1).
TIL 1383I TCR complexes were tethered to the CM5 sensor surface and HLA-A2-tyrosinase complexes were injected over the surface (Methods and Fig. 5).

The solution (3D) affinity of the TIL 1383I TCR was $13 \mu \mathrm{M}$ (Fig. 5a and text).

The original article can be found online at https://doi. org/10.1007/s00262-017-2073-0.

\section{Tamson Moore}

tamoore@luc.edu

1 Department of Surgery, Loyola University Chicago, $2160 \mathrm{~S}$. 1st Avenue, Maywood, IL 60153, USA

2 Department of Medicine, Loyola University Chicago, 2160 S. 1st Avenue, Maywood, IL 60153, USA

3 Department of Pathology, Loyola University Chicago, 2160 S. 1st Avenue, Maywood, IL 60153, USA

4 Present Address: Forefront Dermatology, 801 York St, Manitowoc, WI 54220, USA

5 O. Wayne Rollins Research Center, Emory University, Room 3127, 1510 Clifton Road NE, Atlanta, GA 30322, USA

6 Department of Chemistry \& Biochemistry and the Harper Cancer Research Institute, University of Notre Dame, 251 Nieuwland Science Hall, Notre Dame, IN 46656, USA

7 Present Address: Abbvie, 1 North Waukegan Road, North Chicago, IL 60064, USA
8 Lentigen Technology Inc, A Miltenyi Biotec Company, 910 Clopper Road Suite 200S, Gaithersburg, MD 20878, USA

9 Hollings Cancer Center, Medical University of South Carolina, 86 Jonathan Lucas St, Charleston, SC 29425, USA

10 Dartmouth-Hitchcock, Norris Cotton Cancer Center, One Medical Center Dr, Lebanon, NH 03756, USA

11 Department of Microbiology, and Immunology, Loyola University Chicago, 2160 S. 1st Avenue, Maywood, IL, USA

12 Present Address: Bluebird Biology, 60 Binney St., Cambridge, MA 02142, USA

13 Present Address: Lurie Comprehensive Cancer Center, Department of Dermatology, Northwestern University at Chicago, Room 5-113, 303 East Superior Street, Chicago, IL 60611, USA 